\title{
MULTILINEAR DIRECT AND REVERSE STOLARSKY INEQUALITIES
}

\author{
IOSIF PINELIS
}

Abstract. For any nonnegative measurable function $f:[0,1] \rightarrow \mathbb{R}$ and any $a>0$, let $Q(f, a)$ denote the Stolarsky transform of $f$, equal to $\int_{0}^{1} f\left(x^{1 / a}\right) d x$. Let $S_{n}$ stand for the set of all permutations of the set $\{1, \ldots, n\}$. It is shown that the function

$$
(0, \infty)^{n} \ni \mathbf{a}=\left(a_{1}, \ldots, a_{n}\right) \longmapsto \mathcal{Q}(\mathbf{a}):=\sum_{\sigma \in S_{n}} \prod_{i=1}^{n} Q\left(f_{\sigma(i)}, a_{i}\right)
$$

is Schur-convex if the functions $f_{1}, \ldots, f_{n}$ are nonnegative and nondecreasing and Schur-concave if $f_{1}, \ldots, f_{n}$ are nonnegative and nonincreasing. Necessary and sufficient conditions for the strict Schur convexity and concavity are given.

Similar results are obtained for certain "direct" and "reverse" extensions of the Stolarsky transform to measures.

Mathematics subject classification (2000): 26D15, 26B25, 26 A48.

Key words and phrases: Stolarsky inequality, majorization, Schur convexity.

\section{REFERENCES}

[1] D. H. LuecKing, Without commensurability, Amer. Math. Monthly 1 (1992), 668.

[2] A. W. Marshall AND I. OlKIN, Inequalities: Theory of Majorization and its Applications, Academic, New York, 1979.

[3] R. F. MuIRHEAD, Some methods applicable to identities and inequalities of symmetric algebraic functions of $n$ letters, Proc. Edinburgh Math. Soc. 21 (1903), 144-157.

[4] J. PEČARIĆ, A reverse Stolarsky inequality, Amer. Math. Monthly 101 (1994), 565-567.

[5] K. B. StOlarsky, From Wythoff's Nim to Chebyshev's inequality, Amer. Math. Monthly 98 (1991), 889-900. 